DESY $-96-36$

CERN-TH/96-83

ANL-HEP-PR-96-23

EDINBURGH-96-2

\title{
Associated Jet Production at HERA
}

\author{
J. Bartels ${ }^{1}$, V. Del Duca ${ }^{2}$, A. De Roeck ${ }^{3}$, D. Graudenz ${ }^{4}$, M. Wüsthoff ${ }^{5, a}$ \\ ${ }^{1}$ II. Institut für Theoretische Physik, Universität Hamburg, D-22761 Hamburg, Germany. \\ 2 Dept. of Physics and Astronomy, University of Edinburgh, Edinburgh EH9 3JZ, Scotland, U.K. \\ 3 Deutsches Elektronen Synchrotron DESY, D-22603 Hamburg, Germany. \\ ${ }^{4}$ CERN, Theory Division, 1211 Geneva 23, Switzerland. \\ 5 Argonne National Laboratory, 9700 South Cass Avenue, IL 60439, USA. \\ ${ }^{a}$ Partly supported by the U.S. Department of Energy, Division of High Energy Physics, Contract \\ W-31-109-ENG-38.
}

\begin{abstract}
We compare the BFKL prediction for the associated production of forward jets at HERA with fixed-order matrix element calculations taking into account the kinematical cuts imposed by experimental conditions. Comparison with H1 data of the 1993 run favours the BFKL prediction. As a further signal of BFKL dynamics, we propose to look for the azimuthal dependence of the forward jets.
\end{abstract}


1. Introduction: The BFKL [1] Pomeron has recently gained much interest, in particular in connection with the rise of $F_{2}$ at small Bjorken- $x\left(x_{B}\right)$ observed at HERA. Since it is generally believed that, at small $x, F_{2}$ is driven by the gluon density in the proton, it is tempting to use the BFKL evolution equation for calculating the input distribution $g\left(x, Q_{0}^{2}\right)$. However, such quantitative BFKL-based predictions for $x g\left(x, Q^{2}\right)$ are plagued by theoretical difficulties, and it is therefore important to look for other signals of the BFKL dynamics. A proposed clean "BFKL footprint" at HERA is the measurement of inclusive jets in deep inelastic scattering in the forward direction [2] with longitudinal momentum close to the proton, i.e. the fractional momentum of the jet $x_{j e t} \gg x_{B}$, and transverse momentum, $k_{t}$, of the order of the virtual photon mass $Q^{2}$. Analytic calculations [3] and recent data from H1 [4] show encouraging agreement, but the comparison still suffers from several shortcomings. First, the existing theoretical estimates of the cross section do not take into account the full experimental cuts, which are actually used in the data analysis. Secondly, in order to provide a solid "proof of existence" of the BFKL Pomeron, it is necessary to compare the data not only with the BFKL calculation but also with the fixed-order jet production cross section based upon QCD matrix elements. The BFKL prediction - which, as a result of gluon production between the forward jet and the current jet, rises as a function of $1 / x_{B}$ - is expected to lie above the matrix element calculation, and the data will discriminate between the two predictions. A third obstacle in verifying the BFKL prediction is the lack of hadronization in the theoretical analysis: Monte Carlo studies indicate that, for this type of measurements, hadronization may not be too strong an effect, but there does remain a serious uncertainty. An improvement in this direction might be achieved by the development of a BFKL-based Monte Carlo program. Further evidence for the BFKL Pomeron can be obtained by looking into the azimuthal dependence of the forward jet cross section: in the HERA reference frame BFKL dynamics predicts [5] that, with increasing rapidity difference between the forward jet and the current jet, the forward jet "forgets" about the azimuthal direction defined by the outgoing electron, and the cross section becomes $\Phi$-independent. This decorrelation is, again, a result of the radiation of gluons between the forward jet and the current jet. Correspondingly, in the fixed-order matrix element calculation one expects the $\Phi$ dependence to persist, even for large rapidity intervals. For the Mueller-Navelet [6] jet production at hadron colliders this decorrelation effect has been investigated [0], and it has been demonstrated that such a measurement might give a clear signal for the BFKL Pomeron between the two jets. For the analysis of experimental data from HERA an analytic calculation was performed recently [5]. A numerical analysis, however, has not been done yet.

In the present paper we will first improve on the comparison between the cross section formula and the data. Based upon the analytic expressions given in Ref. [3] we compute the cross section, taking into account the full experimental cuts used in the recent $\mathrm{H} 1$ analysis [4]. We then make use of the fixed-order matrix element calculations for the two- and three-jet cross section [B] and compute, with the same phase space cuts, the numerical predictions. Both calculations are then compared with the data. In the final part of this paper we suggest a new measurement of the azimuthal decorrelation to reveal the BFKL Pomeron. We discuss and present a numerical analysis of the decorrelation in the azimuthal angle $\Phi$, both in the BFKL framework and within the fixed-order jet cross section calculation.

2. Experimental Considerations: The study of associated jet production is an experimental challenge. It was shown in Ref. [3] that the requirement of $x_{j e t} / x_{B}$ to be large results in typical jet angles of a few degrees with respect to the proton direction, also termed forward direction. Due 
to the unavoidable beam-pipe hole for the detectors at the ep collider HERA, the acceptance is limited to jets with an angle larger than, for example, $6^{\circ}$ in the present $\mathrm{H} 1$ detector. At smaller angles the jets are insufficiently contained in the detector and the experimental separation from proton remnant fragments is difficult. The angular requirement leads to maximally reachable $x_{j e t^{-}}$ values of about 0.1 . Furthermore, due to the limited amount of data, a compromise has to be found for the condition $k_{t}^{2} / Q^{2} \sim 1$. This condition is essential since it defines a kinematical environment where no ambiguity between the DGLAP evolution and the BFKL Pomeron exists. In practice an interval is defined around this central value of $k_{t}^{2} / Q^{2}$. The H1 collaboration has made a first measurement of the associated or forward-jet production in deep inelastic scattering 沟. The selections are guided by the limited statistics $\left(300 \mathrm{nb}^{-1}\right)$ and can be refined for future large-statistics samples. In this paper we adopted similar selection criteria. Deep inelastic events are selected by requiring the energy and angle of the scattered electron to be larger than $12 \mathrm{GeV}$ and less than $173^{\circ}$, respectively. The first requirement avoids regions with large contributions of radiative DIS events and experimental background due to misidentified photoproduction events, while the second matches the acceptance of the calorimeter that detects the scattered electron. In order not to confuse the forward jet with the one at the top of the ladder, the requirement $y>0.1$ was imposed to ensure that the jet of the struck quark is well within the central region of the detector and is expected to have a jet angle larger than $60^{\circ}$. Experimentally a cone algorithm is used to find jets, requiring an $E_{t}$ larger than $5 \mathrm{GeV}$ in a cone of radius $R=\sqrt{\Delta \eta^{2}+\Delta \phi^{2}}=1.0$ in the space of pseudo-rapidity $\eta$ and azimuthal angle $\Phi$ in the HERA frame of reference. For the jet selection we consider two sets of cuts, the first set (selection A) matches the published data, while the second set (selection B) could be used when more statistics becomes available and the systematics gets under better control. For selection A jets are accepted as forward jets if $x_{\text {jet }}>0.025,0.5<p_{\text {jet }}^{2} / Q^{2}<4,6^{\circ}<\theta_{\text {jet }}<20^{\circ}$ and $p_{j e t}>5 \mathrm{GeV}$, where $\theta_{\text {jet }}$ is the forward jet angle, $p_{\text {jet }}$ is the transverse momentum of the jet, in approximation of $k_{t}$. Selection B accepts forward jets if $x_{j e t}>0.035,0.5<p_{\text {jet }}^{2} / Q^{2}<2$, $7^{\circ}<\theta_{j e t}<20^{\circ}$ and $p_{\text {jet }}>3.5 \mathrm{GeV}$. These selection criteria allow a study of the cross section of forward jet production in the region $Q^{2} \approx 20 \mathrm{GeV}^{2}$ and $1 \times 10^{-4}<x_{B}<2 \times 10^{-3}$. Hence the ratio $x_{j e t} / x_{B}$ is always larger than 10.

3. The BFKL Approach: The BFKL approach makes a prediction for the high-energy $\left(x_{B} \ll\right.$ $\left.x_{j e t}\right)$ behaviour of the cross section for the process $g \rightarrow g+(n g)+q \bar{q}$ or $q \rightarrow q+(n g)+q \bar{q}$. Consequently, the first nonvanishing contributions appear in the $(3+1)$-jet matrix elements ing from graphs with one gluon in the $t$-channel (for the $(2+1$ )-jet processes $q \rightarrow q g$ and $g \rightarrow q \bar{q}$, there are no contributions with gluons in the $t$-channel, and therefore their dependence on $x_{j e t} / x_{B}$ is power-suppressed). A generic diagram is shown in Fig. 1a.

Using the gauge condition $(q+x p) \cdot A=0$, where $q$ and $p$ are the four-momenta of the virtual photon and proton, respectively, the diagrams corresponding to the two processes $g \rightarrow g q \bar{q}$ and $q \rightarrow q q \bar{q}$ have to be calculated. The incoming parton is always scattered into the forward region, well separated in rapidity from the photon region. Moreover, the two diagrams differ only in their colour content, since the coupling of the $t$-channel gluon to the incoming parton (gluon or quark) is of eikonal type. Due to the large rapidity gap given by $\ln \left(x_{j e t} / x_{B}\right)$ the probability to emit more gluons is increased, for the smallness of the strong coupling constant $\alpha_{s}$ is compensated by the large logarithm $\ln \left(x_{j e t} / x_{B}\right)$. This means that higher-order corrections become relevant, and in the end all diagrams with an arbitrary number of $s$-channel gluons have to be considered. A generic

\footnotetext{
${ }^{1}$ The notation " $(n+1)$ " stands for $n$ jets in the current fragmentation region and one jet in the target fragmentation region, the latter consisting of the proton remnants.
} 


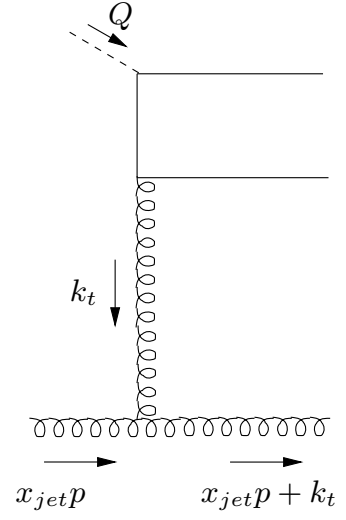

(a)

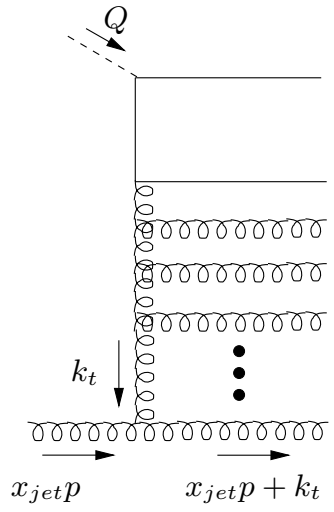

(b)

Figure 1: Generic diagrams for the process (a) $g \rightarrow g+q \bar{q}$ and (b) $g \rightarrow g+(n g)+q \bar{q}$. Diagrams with an incident quark (not shown) have been considered as well.

diagram of this process is shown in Fig. 1b. Their resummation is performed in terms of the BFKL equation [1] and leads to the following result for the one-parton inclusive cross section [3]:

$$
\begin{aligned}
\frac{d \sigma}{d x_{j e t} d k_{t}^{2} d y d Q^{2}}= & \frac{\alpha_{e m}^{2}}{2 y Q^{4}}\left\{\left[1+(1-y)^{2}\right] x_{B} W_{t}^{(0)}+4(1-y) x_{B} W_{l}^{(0)}\right\} \\
& \cdot \frac{3 \alpha_{s}}{\pi k_{t}^{4}}\left\{g\left(x_{j e t}, k_{t}^{2}\right)+\frac{4}{9} \sum_{f} q_{f}\left(x_{j e t}, k_{t}^{2}\right)\right\}
\end{aligned}
$$

with

$$
\begin{array}{r}
x_{B} W_{t}^{(n)=} \sum_{f} Q_{f}^{2} \alpha_{s} \sqrt{k_{t}^{2} Q^{2}} \frac{\pi}{4} \int_{0}^{\infty} d \mu \frac{\frac{9}{4}+\mu^{2}}{1+\mu^{2}} \frac{\sinh (\mu \pi)}{\mu \cosh ^{2}(\mu \pi)} \\
\cdot\left(\frac{x_{j e t}}{x_{B}}\right)^{\omega(n, \mu)} \cos \left(\mu \ln \left(\frac{k_{t}^{2}}{Q^{2}}\right)\right), \\
x_{B} W_{l}^{(n)=} \sum_{f} Q_{f}^{2} \alpha_{s} \sqrt{k_{t}^{2} Q^{2}} \frac{\pi}{4} \int_{0}^{\infty} d \mu \frac{\frac{1}{4}+\mu^{2}}{1+\mu^{2}} \frac{\sinh (\mu \pi)}{\mu \cosh ^{2}(\mu \pi)} \\
\cdot\left(\frac{x_{j e t}}{x_{B}}\right)^{\omega(n, \mu)} \cos \left(\mu \ln \left(\frac{k_{t}^{2}}{Q^{2}}\right)\right)
\end{array}
$$

where

$$
\omega(n, \mu)=\frac{3 \alpha_{s}}{\pi}\left\{2 \psi(1)-\psi\left(\frac{n+1}{2}+i \mu\right)-\psi\left(\frac{n+1}{2}-i \mu\right)\right\} .
$$

Here $n \geq 0$ is an integer, and $W_{t}$ and $W_{l}$ refer to the transverse and the longitudinal parts of the hadronic tensor $W^{\rho \sigma}$. The transverse momentum of the jet and its longitudinal energy fraction are 
given by $k_{t}$ and $x_{j e t}$. The first-order running coupling constant was used with $k_{t}$ as scale. The main features of the BFKL prediction, in particular the power-like increase at small $x_{B}$, can immediately be read off from Eqs. (2) and (3), making use of the fact that the integrand dominates at $\mu=0$. For $\mu=0$ and $n=0$ one finds $9 / 2$ for the ratio of the transverse and the longitudinal part of the cross section. For large $\ln \left(x_{j e t} / x_{B}\right)$ the BFKL power is obtained from $\omega(0,0)$. Before we discuss our numerical results based upon this BFKL formula, we briefly turn to the fixed-order matrix elements.

4. Fixed-Order Matrix Elements Calculations: In order to compare the BFKL results with a fixed-order calculation based on exact matrix elements, we study the contributions for the production of two and three partons on the Born level, giving rise to $(2+1)$ - and $(3+1)$-jet events. We use the PROJET Monte Carlo program [9], where the cross sections from Ref. [8] are implemented in the modified JADE jet-definition scheme 2 . Two partons can be produced in the parton-level processes $q \rightarrow q g$ and $g \rightarrow q \bar{q}$; for three partons there are the processes $q \rightarrow q g g, g \rightarrow q \bar{q} g$ and $q \rightarrow q q \bar{q}$. These matrix elements suffer from infrared and collinear singularities, which are excluded by means of suitable cuts on the parton momenta. This is done by requiring that the invariant mass squared $s_{a b}=2 p_{a} p_{b}$ be larger than some mass scale $M_{c u t}^{2}=c W^{2}, c$ being the jet cut parameter and $W^{2}$ the squared invariant mass of the hadronic final state; $a$ and $b$ are set to $q, \bar{q}, g$ and $r$, where $p_{r}$ is the momentum of the proton remnant jet. All matrix elements except those from the class $g \rightarrow q \bar{q} g$ are singular if any of the invariants goes to zero. The matrix element $g \rightarrow q \bar{q} g$ is not singular for $s_{q \bar{q}}=0$, because of the additional propagator of the quark line. In the following numerical study, we have used the same cuts for the forward jet as those that are applied by the H1 collaboration in their BFKL study. The parton density used is the CTEQ3 leading-order parametrization.

5. Numerical Results: Numerical results for both approaches are shown in Fig. 2. Let us begin with the BFKL approach. The plots depicted in Fig. 2a were evaluated by integrating the differential cross section within the given cuts (see above). The forward jet selection A is used. Two cases are considered:

(i) The fixed-order but high energy asymptotic expression for the two processes $q \rightarrow q+q \bar{q}$ and $g \rightarrow g+q \bar{q}$ (lower two curves in Fig. 2a). These cross sections are evaluated in the BFKL-type high energy approximation, and from Eqs. (2) and (3) they are obtained by simply setting $\omega(n, \mu)=0$.

(ii) The full BFKL result according to Eqs. (2) and (3) (the upper two curves in Fig. 2a). Only light quarks $(u, d$, and $s)$ are included. The curves were scaled by $20 \%$ to approximately include the effect of charm production, in accord with recent data [1].

Case (i) shows good agreement with the results of the second approach, the full matrix element calculations (Fig. 2b, see below). The full BFKL approach, however, scales up the normalization roughly by a factor $\left(x_{j e t} / x_{B}\right)^{0.5}$ and reveals a much steeper shape in the $x_{B}$-distribution. In comparison with data, it is in particular the shape of the cross section which is an important test for the presence of BFKL. The absolute normalization suffers from some uncertainty as discussed below. Figure 2c shows the same calculation for the second set of kinematical cuts.

\footnotetext{
${ }^{2}$ Another calculation of $(3+1)$-jet cross sections can be found in Ref. [10]. It should be noted that the JADE jet-definition scheme is different from the cone scheme employed in the experimental analysis 4 .
} 


\section{$\sigma[p b]$}

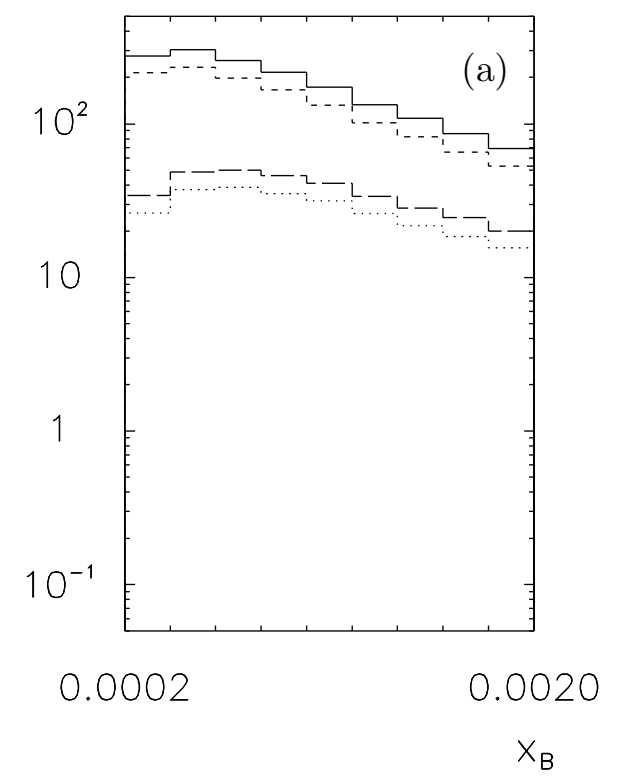

$\sigma[p b]$

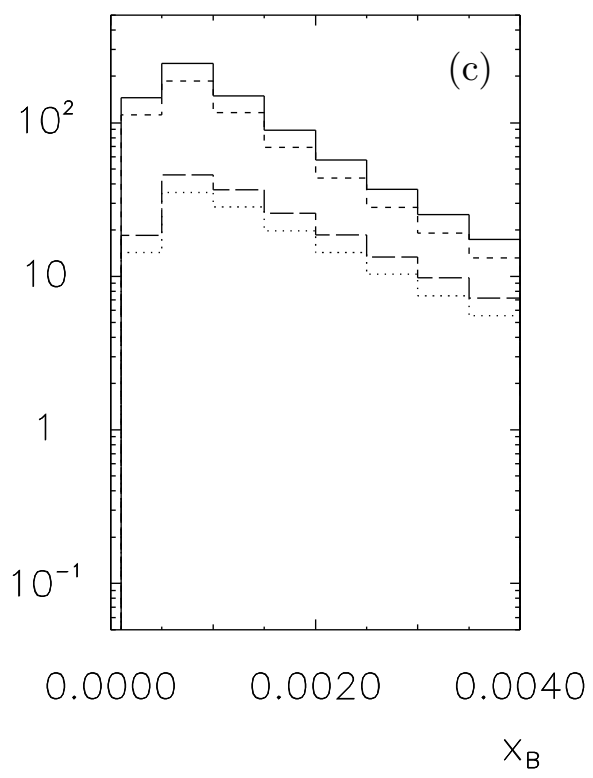

$\sigma[p b]$

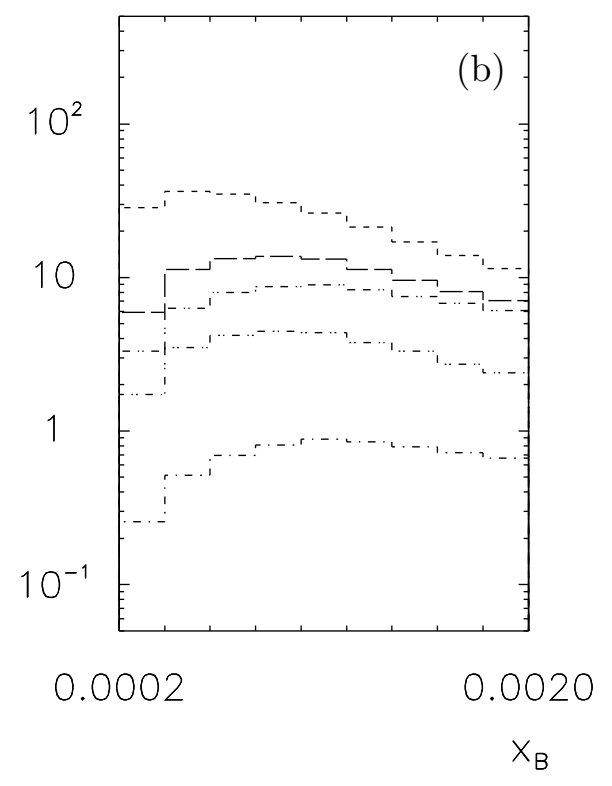

$\sigma[p b]$

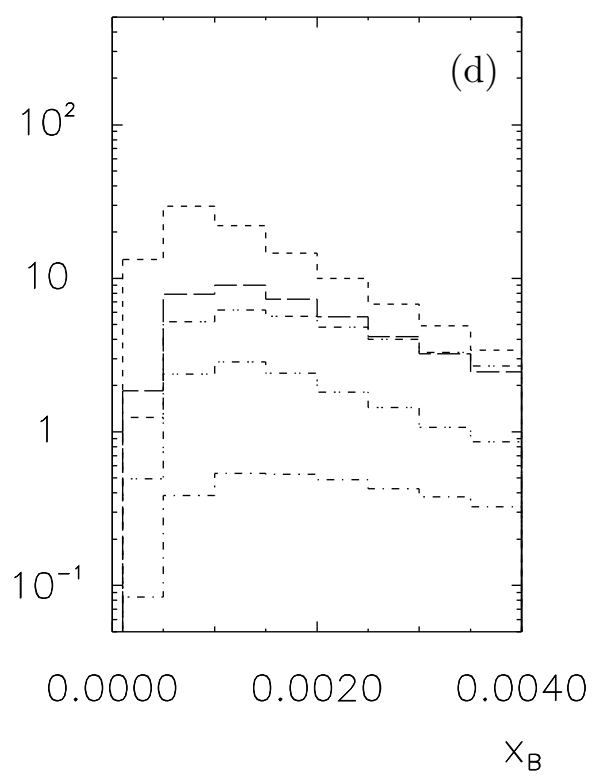

Figure 2: (a) $x_{B}$ dependence of the cross sections from the BFKL calculation (incoming quark and gluon (_) and incoming gluon only (- - -)) and from an approximate analytical calculation of the three-parton matrix elements (incoming quark and gluon ( - - ) and incoming gluon only $(.)$.$) . (b) x_{B}$ dependence of the cross sections from the exact fixed-order matrix elements, with any of the partons as the forward jet. $g \rightarrow q \bar{q} g$ integrated over the full range of the invariant $s_{q \bar{q}}(---)$; the same, but with the additional invariant mass cut $s_{q \bar{q}}>M_{\text {cut }}^{2}(--) ; q \rightarrow q g g$ $(-\cdot-)$ and $q \rightarrow q q \bar{q}(-\cdot \cdot)$, with the cut $s_{A B}>M_{\text {cut }}^{2}$ on all invariants; sum of $q \rightarrow q g$ and $g \rightarrow q \bar{q}$ ( - - ..), with the cut $s_{A B}>M_{\text {cut }}^{2}$ on all invariants. The jet cut parameter is $c=0.005$. The distributions (c) and (d) are the same as (a) and (b), but for the jet cuts set $B$ and calculated for $10^{-4}<x<4 \times 10^{-3}$. 


\section{$\sigma[p b]$}

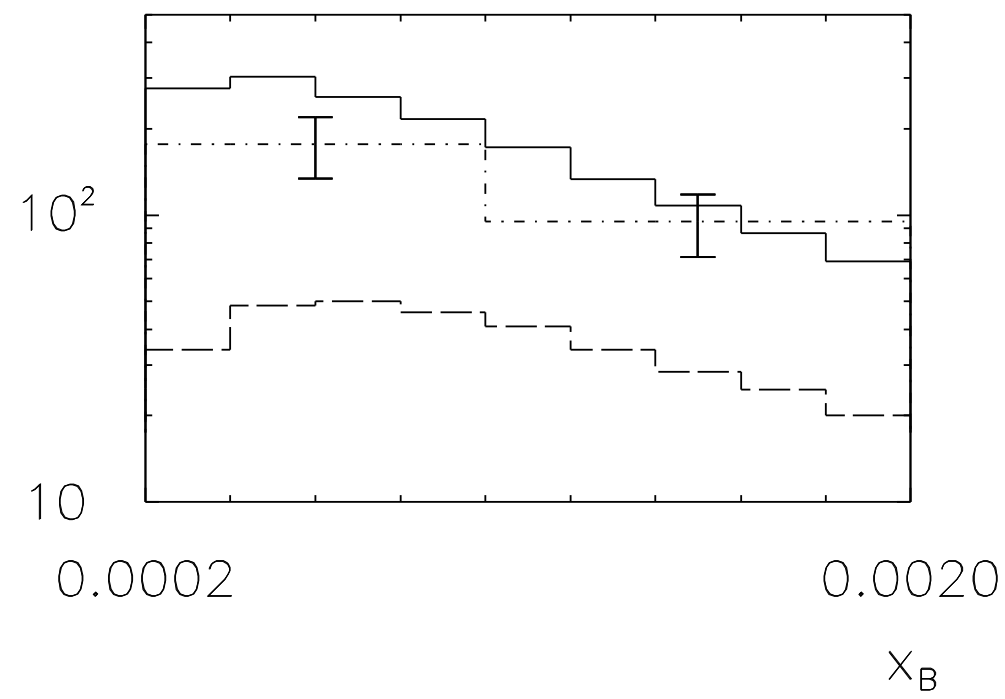

Figure 3: Comparison of the BFKL calculation and the approximate analytical calculation of the three-parton matrix elements, shown in Fig. 2a, compared with the data of the H1 experiment [4].

Next we turn to the second approach, the fixed-order matrix element calculation. The results for the $x_{B}$-dependence are shown in Figs. $2 \mathrm{~b}$ and $2 \mathrm{~d}$ for the jet selections $\mathrm{A}$ and $\mathrm{B}$, respectively. As a result of the applied cut, the contribution from the $(2+1)$-jet matrix element is small. The $(3+1)$ jet contribution $q \rightarrow q g g$ is small as well; the quark densities are small compared with the gluon densities, and the process is not a $t$-channel process. The $q \rightarrow q q \bar{q}$ contribution is larger, because in this class of diagrams there is a $t$-channel gluon which enhances the cross section for forward-jet production. The $(3+1)$-jet cross section is dominated by the process $g \rightarrow g q \bar{q}$. The gluon density is large, and there are large contributions from the diagrams with a $t$-channel gluon. In order to compare the full fixed-order matrix element to the BFKL prediction, it is, in principle, necessary to calculate a cross section that is inclusive in all particle momenta except for the momentum of the forward jet. In the present version of PROJET, such a study is not feasible. However, it is possible to remove the cut $s_{q \bar{q}}>M_{c u t}^{2}$ for the process $g \rightarrow g q \bar{q}$ (see the above discussion). Relaxing this cut increases the cross section considerably. The shape of the $x_{B}$ distribution compares well with that of the approximate analytic calculation for the process $g \rightarrow g q \bar{q}$ (see above), whereas for the full BFKL result (Fig. 2a) the slope in $x_{B}$ is somewhat steeper. It should be noted that the cross section from the exact matrix elements are calculated from a modified JADE scheme and are less "inclusive" than those from the approximate analytic calculation; a direct comparison of the magnitude is therefore not possible.

Finally, we compare the analytic predictions with the H1 data. Because of the JADE jet definition scheme, the fixed-order matrix elements are not quantitatively comparable with the data based on the cone scheme. Moreover PROJET treats the production of charm quarks in the massless approximation. The analysis presented in Ref. [4 has used only two $x_{B}$-bins, and the two values are shown 
in Fig. 3. Obviously, the data are higher than the analytic fixed-order results, indicating that gluon production, which is responsible for the rise of the BFKL Pomeron, is present. On the other hand, the data points lie very close to the BFKL prediction. This looks very encouraging and could be interpreted as the first sign of the presence of the BFKL Pomeron at HERA. However, we feel that several remarks are in order. First, for the analogous process at hadron colliders (Mueller-Navelet jets) a comparison of a next-to-leading order QCD matrixelement calculation with the BFKL approximation has shown [12] that the latter one overestimates the available phase space. A more accurate treatment of the forward jet production, therefore, would most likely tend to lower the BFKL prediction. A similar effect has to be expected also in the forward-jet production at HERA. Secondly, our treatment of the charm contribution is clearly unsatisfactory, and a more accurate treatment of the charm mass has to be included in our calculation. Finally, as we have mentioned in the beginning, also hadronization which is not included in any of our calculations may have some effect. We therefore feel that we have to be cautious in drawing too strong conclusions. It seems safe to say that - within the accuracy to which the data can be compared with our calculations the measurement of forward jets does not agree with the approximate fixed-order matrix element calculation, and the comparison with the BFKL prediction is very encouraging. However, there may be some effects, which can distort this good agreement.

6. Angular decorrelation: Recently [5] the azimuthal dependence of the jet production cross section has been calculated ${ }^{3}$. The angle $\Phi$ is defined in the HERA frame as the angle between the transverse momenta of the outgoing lepton and the forward jet, and the formula for the cross section reads:

$$
\begin{aligned}
& \frac{d \sigma}{d x_{j e t} d k_{t}^{2} d \Phi d y d Q^{2}}= \frac{\alpha_{e m}^{2}}{2 y Q^{4}}\left\{\left[1+(1-y)^{2}\right] x_{B} W_{t}^{(0)}+4(1-y) x_{B} W_{l}^{(0)}\right. \\
&\left.-2(1-y) \cos (2 \Phi) x_{B} W_{l}^{(2)}\right\} \\
& \cdot \frac{3 \alpha_{s}}{2 \pi^{2} k_{t}^{4}}\left\{g\left(x_{j e t}, k_{t}^{2}\right)+\frac{4}{9} \sum_{f} q_{f}\left(x_{j e t}, k_{t}^{2}\right)\right\} .
\end{aligned}
$$

$W_{l}^{(2)}$ is given by Eq. (3) with $n=2$. For small $1 / x_{B}$, Eq. (5) predicts a maximum of the cross section at $\Phi=\pi / 2$. When $1 / x_{B}$ gets large, we expect in the BFKL prediction the $\Phi$-dependence to disappear. In Eq. (5) this effect follows from the difference of the powers $\omega(0,0) \simeq 0.50$ and $\omega(2,0) \simeq-0.22$. With increasing $x_{j e t} / x_{B}$, the angular dependent part is suppressed whereas all other parts increase.

Numerical results are shown in Fig. 4 . In the BFKL case (Fig. 4a) the $\Phi$-dependence is completely washed out when $x_{B}$ becomes small (horizontal line in Fig. 4a). This is in complete agreement with the theoretical expectation. For the approximate fixed-order process $g \rightarrow g q \bar{q}$, on the other hand, the cross section is not flat. It has a clear maximum at $\Phi=\pi / 2$ and is found to be rather insensitive to the choice of the $x_{B}$ bin (not shown). Figure 4c shows the same calculations as Fig. 4a, for the jet selection set B.

For comparison, we also show the results of the fixed-order matrix element calculation (Fig. 4b).

\footnotetext{
${ }^{3} \mathrm{~A}$ somewhat different type of azimuthal dependence has been studied in Ref. [13].
} 

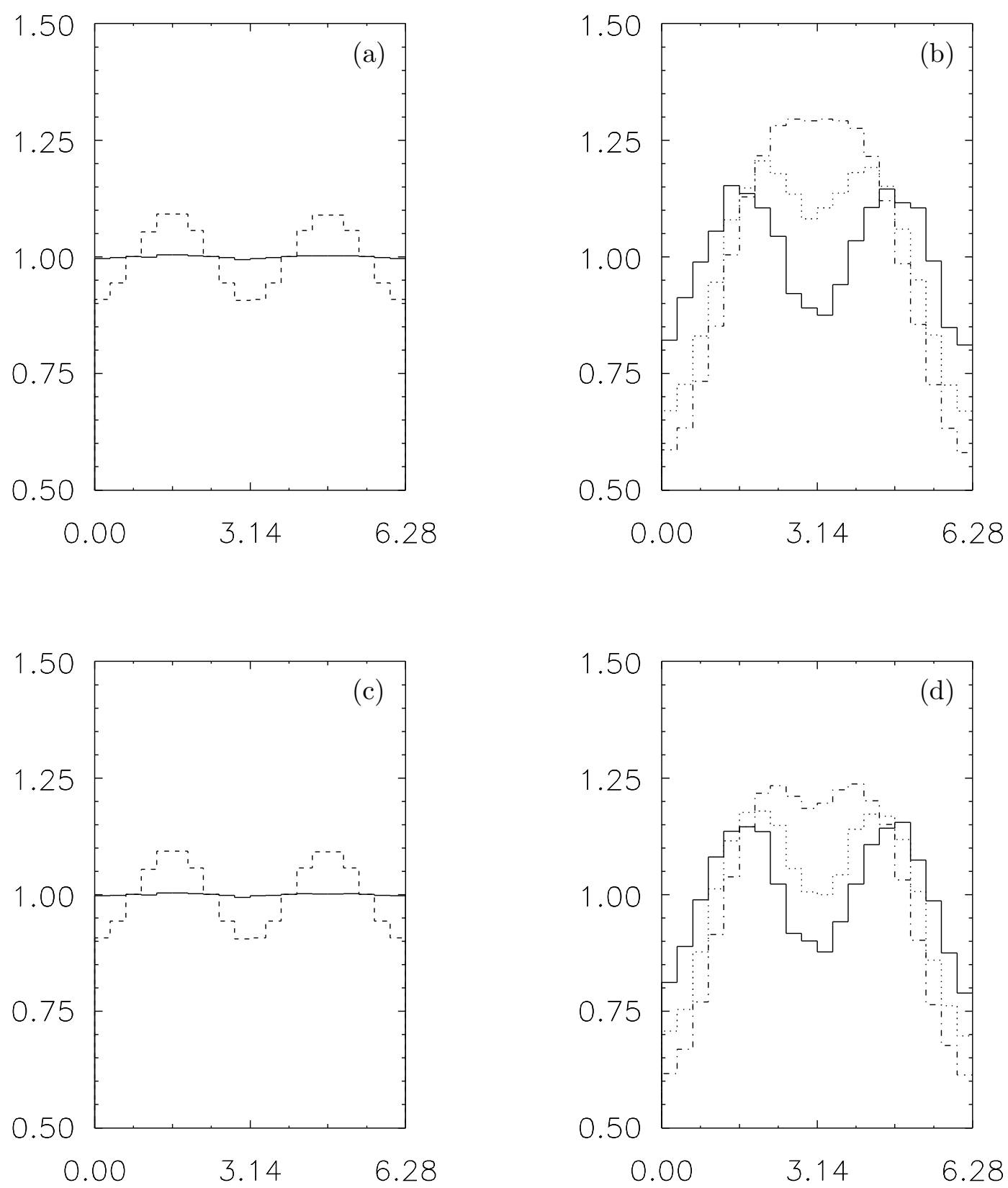

Figure 4: Dependence on the difference $\Phi$ of the azimuthal angles of the outgoing lepton and the forward jet, normalized to a common average. (a) BFKL result, the bin in $x_{B}$ is $\left[0.2 \times 10^{-3}, 0.4 \times\right.$ $10^{-3}$ ] (- ). Approximative analytical Born result, the bin in $x_{B}$ is $\left[0.2 \times 10^{-3}, 0.4 \times 10^{-3}\right]$ $(-\mathbf{-})$. (b) Fixed-order result, the bins in $x_{B}$ are given by $\left[0.2 \times 10^{-3}, 0.4 \times 10^{-3}\right](-)$, $\left[1.8 \times 10^{-3}, 2.0 \times 10^{-3}\right](\cdots)$ and $\left[3.4 \times 10^{-3}, 3.6 \times 10^{-3}\right](-\cdots)$. The jet cut parameter $c=0.005$. The matrix element is $g \rightarrow q \bar{q} g$, integrated over the full range of the invariant $s_{q \bar{q}}$, where any of the partons may be the forward jet. The distributions (c) and (d) are the same as (a) and (b), but for the jet cuts set $B$. 
We have used the jet cut $M_{c u t}^{2}=c W^{2}$ with $c=0.005$. The curve shown in Fig. $4 \mathrm{~b}$ belongs to the process $g \rightarrow g q \bar{q}$, integrated over the full range of the invariant $s_{q \bar{q}}$. The forward jet may belong to any of the partons. At smaller $x_{B}$, there is a tendency to enhance the maximum of the distribution at $\Phi=\pi / 2$ and $\Phi=3 \pi / 2$, similar to the BFKL Born calculation in Fig. 4a. There is a clear difference with the full BFKL prediction in Fig. 4a. At larger $x_{B}$, on the other hand, the full matrix element calculation does not agree very well with the BFKL Born calculation. Figures $4 \mathrm{c}$ and $4 \mathrm{~d}$ show the results for selection $\mathrm{B}$ of the jet cuts.

The present detectors at HERA allow for jet angular measurements with a resolution of about $5^{\circ}-10^{\circ}$ for jets with an $E_{t}$ larger than $5 \mathrm{GeV}$. This opens a window for measuring angular correlations between struck quark and forward jet, or between scattered electron and forward jets. The major background for this measurement is made up of radiative events; due to the changed kinematics at the hadron vertex, these can eject a jet in the forward direction. This background is however expected to be controlled experimentally to a sufficient level to allow for a measurement of better than a $10 \%$ significance level in large data samples. We therefore expect that a measurement of the angular decorrelation and the comparison with theoretical expectations provides a further test of BFKL dynamics.

7. Conclusions: In this paper we have made a comparison for the production cross section of associated jets in the forward direction of the BFKL prediction and of the fixed-order matrix element calculation. Both curves show a clear difference at small $x_{B}$, thus confirming the expectation that this process provides a clean signal of the BFKL Pomeron. Confrontation with the data is encouraging: the data clearly lie above the fixed-order calculation and prove that the radiation of extra gluons is an important effect. The BFKL calculation is very close to the data, which could be the first sign of the presence of the BFKL Pomeron in the HERA data. However, there are several aspects that need further study, in particular the contribution of charm and hadronization effects in the final state. From the theoretical side, there is also the question of the kinematic region in which the BFKL approximation is applicable. We expect that the strongest correction will come from unitarization effects, and we know that first unitarity corrections will lower the BFKL prediction. Hence it may be premature to interpret the data as a proof of the presence of the BFKL Pomeron.

For future measurements we have suggested to look also into the azimuthal dependence of the jet cross section. Comparison with fixed-order calculations show that this provides a new signal for BFKL dynamics, which may help to find further footprints of the BFKL Pomeron at HERA.

\section{References}

[1] E.A. Kuraev, L.N. Lipatov and V.S. Fadin, Sov. Phys. JETP 45 (1977) 199;

Ya.Ya. Balitskij and L.N. Lipatov, Sov. J. Nucl. Phys. 28 (1978) 822.

[2] A.H. Mueller, Nucl. Phys. B (Proc. Suppl.) 18C (1991) 125.

[3] J. Bartels, A. De Roeck and M. Loewe, Z. Phys. C54 (1992) 635;

J. Kwiecinski, A.D. Martin and P.J. Sutton, Phys. Lett. B287 (1992) 254; Phys. Rev. D46 (1992) 921;

W.-K. Tang, Phys. Lett. B278 (1991) 363; 
J. Bartels, M. Besançon, A. De Roeck and J. Kurzhoefer, in Proceedings of the HERA Workshop 1992 (eds. W. Buchmüller and G. Ingelman), p. 203.

[4] H1 Collab., DESY-95-108 and Phys. Lett. B356 (1995) 118.

[5] J. Bartels, V. Del Duca and M. Wuesthoff, DESY preprint in preparation.

[6] A.H. Mueller and H. Navelet, Nucl. Phys. B282 (1987) 727.

[7] V. Del Duca and C.R. Schmidt, Phys. Rev. D49 (1994) 4510;

W.J. Stirling, Nucl. Phys. B423 (1994) 56.

[8] D. Graudenz and N. Magnussen, in Proceedings of the HERA Workshop 1991, DESY (eds. W. Buchmüller and G. Ingelman).

[9] D. Graudenz, PROJET 4.13 manual, Comput. Phys. Commun. 92 (1995) 65.

[10] T. Brodkorb, J.G. Körner, E. Mirkes and G.A. Schuler, Z. Phys. C44 (1989) 415.

[11] A. De Roeck, to appear in Proceedings of the 2nd Epiphany Workshop, Krakow, 1996.

[12] V. Del Duca and C.R. Schmidt, Phys. Rev. D51 (1995) 2150.

[13] A. Askew, D. Graudenz, J. Kwiecinski and A.D. Martin, Phys. Lett. B338 (1994) 92. 